\title{
Editorial
}

Bundesgesundheitsbl 2015 · 58:219

DOI 10.1007/s00103-015-2137-2

Online publiziert: 13. Februar 2015

๑) Springer-Verlag Berlin Heidelberg 2015

Rüdiger Klar · Werner Vach

Department für Medizinische Biometrie und Medizinische Informatik, Universität Freiburg,

Freiburg, Deutschland

\section{Nutzenbewertung bei medizinischen Maßnahmen}

fünf Beiträgen, die die Nutzenbewertung für spezielle Arten von medizinischen Maßnahmen beleuchten. Es folgen zwei Beiträge zu methodischen Aspekten der Nutzenbewertung. Den Abschluss bilden zwei Beiträge, die die Nutzenbewertung aus der Sicht der Gesundheitsökonomie bzw. der Versorgungsforschung und Epidemiologie in einen größeren Rahmen einordnen.

Ein gemeinsames Fazit lässt sich leicht aus allen Beiträgen ablesen: Die Nutzenbewertung ist in Deutschland angekommen. Kein Beitrag stellt die Nutzenbewertung an sich als politisches und/oder wissenschaftliches Instrument in Frage. Dies ist nicht ganz selbstverständlich da in Deutschland „die Entwicklung einer evidenzbasierten Nutzenbewertung eher ,Top-down' als ,Bottom-up erfolgt ist", wie Jürgen Windeler und Stefan Lange in ihrem Beitrag darlegen. Allerdings weisen viele Beiträge darauf hin, dass die Nutzenbewertung noch nicht überall angekommen ist und auch nicht immer unbedingt in der Form, wie sie allen Beteiligten wünschenswert erscheint. Nachholbedarf wird vor allem im Bereich der Medizinprodukte, aber auch bei den operativen Fächern angemeldet. Klärungsbedarf wird gesehen, wenn es um die administrativen Rahmenbedingungen und das Zusammenspiel aller Akteure (Beinlich et al., Windeler und Lange), die Einbettung in einen gesamtgesellschaftlichen Kontext (Kaier und Fetzer, Vach und Gerke), die Methodik (Gonnermann et al., Bullinger et al.) und die Reichweite der Methodik (Wegscheider et al.) geht. Insofern ist abzusehen, dass uns das Thema „Nutzenbewertung" und ihre Umsetzung noch viele Jahre beschäftigen werden.
Ihre

R. Klar

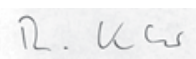

W. Vach

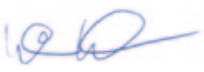

\section{Korrespondenzadresse}

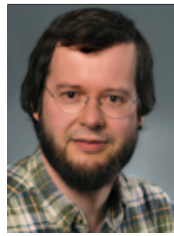

Prof. W. Vach

Department für

Medizinische Biometrie und

Medizinische Informatik

Universität Freiburg

Stefan-Meier-Str. 26

79104 Freiburg

wv@imbi.uni-freiburg.de

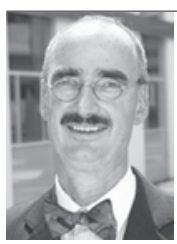

Prof. R. Klar

Department für

Medizinische Biometrie und Medizinische Informatik

Universität Freiburg

Stefan-Meier-Str. 26

79104 Freiburg

ruediger.klar@web.de 